\title{
Atividade da fosfatase alcalina no lavado broncoalveolar de equinos de policiamento montado no Estado do Rio de Janeiro
}

\author{
Alkaline phosphatase activity in bronchoalveolar lavage of police horses in \\ Rio de Janeiro State, Brazil
}

\author{
Maria Luisa Lorêdo Abreu Jorge ${ }^{\mathrm{I}}$ Vanessa Viscardi ${ }^{\mathrm{I}}$ Katia Moreira Silva ${ }^{\mathrm{II}}$ \\ Juliana Nabuco Pereira Otaka ${ }^{\text {II }}$ Nayro Xavier de Alencar ${ }^{\mathrm{II}}$ Rodolpho de Almeida Torres Filho ${ }^{\mathrm{III}}$ \\ Daniel Augusto Barroso Lessa ${ }^{I^{*}}$
}

RESUMO

A utilidade da determinação das atividades enzimáticas no trato respiratório posterior como ferramenta diagnóstica já foi demonstrada em várias espécies. Nesse contexto, este trabalho teve por objetivo determinar a atividade da Fosfatase Alcalina (FAL) no lavado broncoalveolar (LBA) de equinos da Polícia Militar do Estado do Rio de Janeiro, comparando animais sadios com portadores assintomáticos de doença inflamatória das vias aéreas (DIVA). Para tal, foram avaliados 28 animais adultos, machos, sem histórico de doença respiratória nos dois meses anteriores ao estudo, com os resultados dos exames físicos e laboratoriais (FAL sanguínea, hematócrito, leucograma, proteína total e fibrinogênio plasmáticos) dentro dos parâmetros fisiológicos. Os equinos foram divididos em dois grupos de acordo com o resultado da citologia broncoalveolar. A determinação da atividade da FAL foi realizada por meio de espectrofotometria a partir de alíquotas do sobrenadante do LBA preservadas em nitrogênio líquido. Para a estimativa do fluido epitelial pulmonar e da atividade da FAL neste, foi realizada a correção da diluição provocada pelo lavado. Os equinos com contagem diferencial de tipos celulares compatível com DIVA apresentaram atividade de FAL no LBA menor, quando comparados aos animais sadios, podendo essa dosagem ser utilizada como complementação do diagnóstico da DIVA.

Palavras-chave: cavalo, lavado broncoalveolar, fosfatase alcalina, DIVA.

\section{ABSTRACT}

The use of determining the enzymatic activities in the posterior respiratory tract as a diagnostic tool has already been demonstrated in several species. In this context, this paper aims to determine the activity of alkaline phosphatase (ALP) in the bronchoalveolar lavage (BAL) of horses from the Military Police of the State of Rio de Janeiro, comparing healthy animals with asymptomatic carriers of an inflammatory airway disease (IAD). Twenty-eight adult male animals with no history of respiratory diseases in the last two months prior to the study were studied. Physical exam and blood laboratory test results (ALP, hematocrit, leukogram, total protein and plasma fibrinogen) were within physiological parameters. The equines were separated into two groups according to the results of the bronchoalveolar cytology. The determination of $A L P$ was done by spectrophotometry with aliquots of the supernatant of the BAL preserved in liquid nitrogen. To estimate pulmonary epithelial lining fluid and ALP activity, correction of the dilution caused by the lavage was done. The horses with a cell type differential count compatible with IAD presented a lower ALP activity in BAL when compared to healthy animals, therefore this dosage can be used as a complement in the diagnosis of IAD.

Key words: horse, bronchoalveolar lavage, alkaline phosphatase, $I A D$.

\section{INTRODUÇÃO}

A prevalência de doenças inflamatórias pulmonares é alta em equinos (LESSA et al., 2008; GERBER et al., 2009; VAN ERCK, 2009; MAZAN, 2010). Exames clínicos completos, históricos detalhados, bem como exames complementares específicos são de grande relevância para o diagnóstico de doenças respiratórias que evoluem de forma assintomática (VAN ERCK, 2009).

A citologia broncoalveolar é um método sensível para diagnóstico de doenças inflamatórias

'Polícia Militar do Estado do Rio de Janeiro, Esquadrão Escola de Cavalaria, Jardim Sulacap, RJ, Brasil.

"Departamento de Patologia e Clínica Veterinária, Faculdade de Veterinária, Universidade Federal Fluminense (UFF), Rua Vital Brasil Filho 64, 24230-340, Vital Brazil, Niterói, RJ, Brasil. E-mail: misaloredo@ig.com.br. *Autor para correspondência.

II'Departamento de Zootecnia e Desenvolvimento Agrossocioambiental Sustentável, UFF, Niterói, RJ, Brasil. 
não infecciosas das vias aéreas posteriores dos equinos (HOFFMAN, 1999), como a Doença Inflamatória das Vias Aéreas (DIVA). Entretanto, a caracterização citológica dessa enfermidade em animais assintomáticos é dificultada pela grande variação nos valores limítrofes dos tipos celulares, especialmente neutrófilos (FERRO et al., 2002; HOFFMAN, 2008).

O fluido epitelial pulmonar (FEP) contém elementos celulares e moleculares, como por exemplo, enzimas e imunoglobulinas, que podem ser utilizados para a avaliação da integridade do trato respiratório (SILVA et al., 2010). Entretanto, a infusão de solução isotônica para a realização do lavado broncoalveolar (LBA) resulta numa diluição significativa do FEP, sendo necessária a utilização de marcadores como fator de correção. Nesses casos, a ureia é comumente utilizada como marcador de diluição, devido ao seu livre trânsito pelas membranas biológicas (McGORUM et al., 1993).

A fosfatase alcalina (FAL) é uma glicoproteína com função hidrolítica, responsável pela remoção de grupamentos fosfato de vários tipos de moléculas (KANEKO, 1989). Sua atividade sérica é um importante indicador de lesão hepática desde a década de 20 (KALINA et al., 1990). Entretanto, valores alterados da atividade da FAL no soro e em outros fluidos corpóreos podem refletir alterações fisiológicas ou patológicas além daquelas de origem hepática (FERNANDEZ \& KIDNEY, 2007).

Estudos indicam alta atividade da FAL nos pneumócitos do tipo II de secções de tecido pulmonar, bem como em cultivos desse tipo celular. Essa atividade parece estar relacionada com a síntese de compostos fosfolipídicos e proteicos da substância surfactante pulmonar (KALINA et al., 1990). Pesquisadores já demonstraram a importância clínica da utilização da determinação da atividade da FAL no trato respiratório posterior como ferramenta diagnóstica em várias espécies (HENDERSON et al., 1995; CAPELLI et al., 1997; COBBEN et al., 1999; MADEN et al., 2001).

A incorporação de novos métodos que levem à melhoria da eficácia no diagnóstico e, por conseguinte, na terapêutica podem, de forma segura, complementar as informações obtidas pelas técnicas diagnósticas já consagradas. Portanto, é necessário estabelecer e implementar como rotina na clínica médica de equinos, à semelhança do que já acontece para outras espécies, a utilização de outros marcadores de processos inflamatórios pulmonares, como, por exemplo, as enzimas FAL, gama-glutamiltransferase e lactato desidrogenase.
Nesse contexto, este estudo teve por objetivo determinar a atividade da FAL no LBA de equinos adultos, sadios e com DIVA assintomática, utilizados no policiamento montado no Estado do Rio de Janeiro, Brasil.

\section{MATERIAL E MÉTODOS}

Foram avaliados 28 equinos adultos, machos, sem raça definida, com idade entre cinco e 14 anos, pesando em média $460 \mathrm{~kg}$, utilizados em policiamento montado e instrução de montaria, pertencentes à Policia Militar do Estado do Rio de Janeiro (PMERJ), Brasil. Os animais foram mantidos semi-estabulados, em baias de $6 \mathrm{~m}^{2}$ sem cama, de seis às 15 horas e o restante do período mantidos em solário. Foram alimentados com seis $\mathrm{kg}$ animal $^{-1}$ de ração comercial (com $12 \%$ de proteína bruta) e $12 \mathrm{~kg}_{\text {animal }}{ }^{-1}$ de Capim-Angola (Brachiaria mutica), cortado e oferecido no solário, e com água ad libitum. Os animais são submetidos à vermifugação trimestral, vacinação semestral contra influenza, tétano, encefalomielite equina leste e oeste, rinopneumonite; e anual contra raiva. O controle da anemia infecciosa equina é realizado segundo as normas vigentes do Ministério da Agricultura, Pecuária e Abastecimento (MAPA).

Para a seleção dos animais, foi realizado exame físico, com especial ênfase ao exame do sistema respiratório, considerando os valores de referência descritos por HOUSTON \& RADOSTITS (2002) para as funções vitais e por McGORUM et al. (2002) para os resultados da percussão e ausculta pulmonar. Os valores de hematócrito, leucograma, bem como FAL sanguínea, proteína total e fibrinogênio plasmáticos estavam dentro da normalidade. Durante o estudo, todos os equinos apresentaram-se clinicamente sadios, não foram submetidos a tratamentos e nem foram relatados sintomas de doença respiratória nos dois meses anteriores.

Amostras de sangue venoso foram coletadas por meio de punção jugular, utilizando-se tubos a vácuo (Vacutainer $^{\circledR}$ ) com EDTA e agulhas hipodérmicas $21 \mathrm{G}$ para realização do hematócrito, leucograma, proteína plasmática total e fibrinogênio. Esses exames foram executados conforme as metodologias descritas por JAIN (1993). Para a determinação da ureia sérica, o sangue foi colhido em tubos a vácuo sem anticoagulante, no momento da realização do LBA, e mantidos à temperatura ambiente até que ocorresse a coagulação deste. Ato contínuo, as amostras foram centrifugadas e o soro armazenado à temperatura de $-14 \mathrm{a}-18^{\circ} \mathrm{C}$ até o momento das determinações, que não ultrapassou dois meses. A concentração da ureia foi determinada em 
duplicata, por meio de espectrofotometria (Analisador bioquímico semiautomático Bioplus 2000 ${ }^{\circledR}$ ), utilizando-se "kits" Ureia UV Liquiform $\left(\right.$ Labtest $\left.{ }^{\circledR}\right)$.

Para obtenção do LBA, os animais foram sedados com cloridrato de xilazina a $2 \%$ (Coopazine $^{\circledR}$, Coopers Brasil Ltda.), na dosagem de 0,5$1,1 \mathrm{mg} \mathrm{kg}^{-1}$, via intravenosa e contidos com cachimbo. Antes da introdução da sonda, foi realizada a limpeza das narinas com água morna. O trato respiratório a partir da laringe foi anestesiado durante a introdução da sonda para lavagem $\left(\right.$ Bivona $\left.^{\circledR}\right)$, com instilação de cloridrato de lidocaína a 0,5\% (Anestésico Bravet ${ }^{\mathbb{R}}$, Laboratório Bravet). O LBA foi obtido por meio da infusão de $500 \mathrm{~mL}$ de solução salina estéril aquecida a $37^{\circ} \mathrm{C}$, divididos em duas alíquotas de $250 \mathrm{~mL}$, com imediata aspiração e acondicionamento em frascos de vidro, mantidas sob refrigeração e processadas em até quatro horas. Foram consideradas amostras satisfatórias quando recuperados pelo menos $40 \%$ (200mL) do volume total infundido.

Alíquotas de $200 \mu \mathrm{L}$ da suspensão celular do LBA foram submetidas à citocentrifugação a 110 x G por cinco minutos (centrífuga Serocito ${ }^{\circledR}$ modelo 2400 Fanem $^{\circledR}$ ). As lâminas confeccionadas foram fixadas e coradas pelo corante Giemsa. A leitura foi realizada em microscópio óptico com objetiva de imersão de 100X, sendo analisados os tipos celulares durante a contagem de 500 células.

A partir dos resultados da citologia broncoalveolar, dois grupos foram formados: grupo 1, composto por 10 animais com valores citológicos normais, e grupo 2, formado por 18 animais com citologia compatível com DIVA, apresentando percentuais superiores a $2 \%$ de mastócitos e/ou $5 \%$ de neutrófilos e/ ou $1 \%$ de eosinófilos, bastando um desses parâmetros para tal caracterização, conforme estabelecido no consenso de Havemeyer, ratificado por MAZAN (2010).

A determinação da atividade da FAL foi realizada em alíquotas do sobrenadante do LBA, centrifugadas a $800 \times \mathrm{G}$ por cinco minutos (centrífuga Fanem ${ }^{\circledR}$ modelo 206R) e preservadas em nitrogênio líquido $\left(-196^{\circ} \mathrm{C}\right)$. Para dosagem da concentração de ureia, alíquotas do LBA foram centrifugadas com o mesmo protocolo e estocadas entre -14 e $-18^{\circ} \mathrm{C}$. As amostras foram analisadas em duplicata por meio de espectrofotometria, como descrito anteriormente para análise da ureia sérica.

A partir de concentrações previamente conhecidas de ureia no LBA de equinos (McGORUM et al., 1993) e utilizando-se o padrão fornecido pelo fabricante do "kit" em diluições seriadas, foi estabelecida uma equação de regressão linear e sua curva-padrão, utilizadas para cálculo das concentrações nas amostras de LBA, conforme metodologia descrita por SILVA et al. (2010).

O conjunto de informações obtidas foi submetido à análise descritiva dos dados, utilizando o programa computacional SAS ${ }^{\circledR}$ a fim de verificar os pressupostos da análise de variância (teste F), em que se constatou a necessidade de transformar os dados referentes à atividade da FAL no LBA, transformação esta feita pela escala logarítmica na base 10. A análise de variância foi realizada pelo procedimento GLM (General Linear Models) do programa estatístico $\mathrm{SAS}^{\circledR}$, adotando-se o nível de significância de 5\%

\section{RESULTADOS E DISCUSSÃO}

Os animais avaliados neste trabalho com citologia broncoalveolar compatível com DIVA apresentaram atividade da FAL no LBA estatisticamente menor $(\mathrm{P}<0,05)$ do que animais com citologia normal (Tabela 1). A atividade da FAL em LBA é um marcador de lesão celular e de proliferação de células do tipo II em ratos e seres humanos (HENDERSON et al., 1995; CAPELLI et al., 1997). Em estudo com ratos portadores de afecções pulmonares tóxicas agudas, HENDERSON et al. (1995) concluíram que a maior fonte do aumento na atividade de FAL em LBA foram os pneumócitos tipo II.

Em seres humanos, o aumento da atividade da FAL no LBA está relacionado a enfermidades pulmonares intersticiais, fibróticas e pneumopatias de caráter agudo, com ou sem componente infeccioso, mas que produzem lesões significativas de epitélio (CAPELLI et al., 1997; COBBEN et al., 1999; SANCHEZ et al., 2000). Estudos realizados em cães por MADEN et al. (2001) demonstraram aumento na atividade da FAL no LBA em cães acometidos por broncopneumonia, fato não ocorrido em cães com traqueobronquite, indicando a necessidade de enfermidades mais graves para tal aumento.

Se, em equinos, a atividade da FAL está relacionada à função dos pneumócitos do tipo II, à semelhança do que ocorre em outras espécies, é de se

Tabela 1 - Atividade da fosfatase alcalina no fluido epitelial pulmonar em $\mathrm{U} \mathrm{L}^{-1}$ dos grupos experimentais 1 e 2 de equinos da Polícia Militar do Estado do Rio de Janeiro, expressa em média e desvio-padrão. Rio de Janeiro, Brasil, 2011

\begin{tabular}{lll}
\hline & Grupo 1 & Grupo 2 \\
\hline Média & $3245,43^{*}$ & $2041,90^{*}$ \\
Desvio-padrão & 1449,90 & 761,13 \\
\hline
\end{tabular}

*Apresenta diferença estatisticamente significativa $(\mathrm{P}<0,05)$. 
esperar que a atividade da FAL se encontre baixa em animais com quadros de DIVA crônica assintomática, como os observados no grupo 2. Diferentemente do que acontece nas pneumopatias previamente citadas, que cursam com dano epitelial significativo e aumento da atividade de FAL, as lesões teciduais na DIVA são menos graves. VIEL (1986) e MAZAN (2010) descreveram infiltrados de células inflamatórias em mucosas, exsudatos luminais, hiperplasia bronquial e metaplasia de células caliciformes como as lesões encontradas em biópsias bronquiolares de cavalos atletas jovens com inflamação de vias aéreas posteriores. Tais lesões podem não ser suficientes para refletir aumento de atividade de FAL, entretanto, podem afetar a função dos pneumócitos do tipo II com consequente redução na atividade enzimática. Essa relação foi comprovada em estudo de EDELSON et al. (1988), em que a atividade dessa enzima se mostrou reduzida em cultivo de pneumócitos do tipo II de ratos em meio sabidamente desfavorável. Quando essas células, após 48 horas, foram removidas para um meio favorável, houve restabelecimento das funções e consequente aumento da atividade da FAL.

Estudos utilizando a dosagem da atividade de FAL pulmonar em equinos até o momento não foram publicados. Sendo assim, o presente trabalho trata de forma pioneira o comportamento dessa enzima no LBA de equinos sadios e com DIVA assintomática e destaca a importância de ampliar esses estudos para enfermidades pulmonares mais graves nessa espécie, assim como para o estabelecimento de valores fisiológicos da atividade desta enzima no LBA de equinos.

\section{CONCLUSÃO}

Diante dos resultados obtidos neste trabalho, pode-se concluir que a atividade da fosfatase alcalina no lavado broncoalveolar em equinos com doença inflamatória das vias aéreas apresentou-se mais baixa, quando comparada à atividade enzimática nos animais sadios.

A determinação da fosfatase alcalina no lavado broncoalveolar pode ser usada como meio de complementação do diagnóstico da doença inflamatória das vias aéreas em equinos adultos que possuam o mesmo perfil de trabalho, sem queixa clínica e com citologia broncoalveolar compatível com essa enfermidade.

\section{COMITÊ DE ÉTICA}

Este trabalho foi realizado em acordo com os princípios éticos na experimentação animal do Colégio Brasileiro de Experimentação Animal (COBEA) e obteve a aprovação do Comitê de Ética em Pesquisa Animal (CEPA), sob ㄲo 00106/09.

\section{REFERÊNCIAS}

CAPELLI, A. et al. Lung alkaline phosphatase as a marker of fibrosis in chronic interstitial disorders. American Journal of Respiratory and Critical Care Medicine, v.155, n.1, p.249-253, 1997.

COBBEN N.A. et al. Relationship between enzymatic markers of pulmonary cell damage and cellular profile: A study in bronchoalveolar lavage fluid. Experimental Lung Research, v.25, n.2, p.99-111, 1999. doi:10.1080/019021499270321.

EDELSON, J.D. et al. Alkaline phosphatase: a marker of alveolar type II cell differentiation. American Journal of Respiratory and Critical Care Medicine, v.138, n.5, p.1268-1275, 1988. doi:10.1164/ajrccm/138.5.1268

FERNANDEZ, N.J.; KIDNEY, B.A. Alkaline phosphatase: beyond the liver. Veterinary Clinical Pathology, v.36, n.3, p.223233, 2007. doi:10.1111/j.1939-165X.2007.tb00216.x.

FERRO, E. et al. Arterial blood gas analysis in 53 racehorses with diagnosis of small airway inflammatory disease (SAID). Journal of Equine Veterinary Science, v.22, n.4, p.165-168, 2002. doi:10.1016/S0737-0806(02)70142-1.

GERBER, V. et al. IAD in the sport and leisure horse. In: WORLD EQUINE AIRWAYS SYMPOSIUM, 4., 2009, Berne, Switzerland. Proceedings... Berne, Switzerland: WEAS, 2009. p.183-185.

HENDERSON, R.F. et al. Source of alkaline phosphatase activity in epithelial lining fluid of normal and injured F344 rat lungs. Toxicology and Applied Pharmacology, v.134, n.1, p.170-174, 1995.

HOFFMAN, A.M. Bronchoalveolar lavage technique and cytological diagnosis of small airway inflammatory disease. Equine Veterinary Education, v.11, n.6, p.330-336, 1999. doi: 10.1111/j.2042-3292.1999.tb01566.x.

HOFFMAN, A.M. Bronchoalveolar lavage: Sampling technique and guidelines for cytologic preparation and interpretation. Veterinary Clinics of North America: Equine Practice, v.24, n.2, p.423-435, 2008. doi:10.1016/j.cveq.2008.04.003.

HOUSTON, D.M.; RADOSTITS, O.M. O exame clínico. In: RADOSTITS, O. et al. (Eds.). Exame clínico e diagnóstico em veterinária. Rio de Janeiro: Guanabara Koogan, 2002. Cap.6, p.81.

JAIN, N.C. Essentials of veterinary hematology. Philadelphia: Lea and Febiger,1993. 417p.

KALINA, M. et al. Modulation of alkaline phosphatase activity in alveolar type II like cells. Histochemistry, v.95, p.97-103, 1990. Disponível em: <http://www.springerlink.com/content/ r78138073kj077kw/>. Acesso em: 13 nov. 2010.

KANEKO, J.J. Clinical biochemistry of domestic animals. 5.ed. San Diego: Academic, 1989. 932p.

LESSA, D.A.B. et al. Doença Inflamatória das vias aéreas (DIVA) em equinos de policiamento na cidade do Rio de Janeiro, RJ: estudo clínico. Revista Brasileira de Ciência Veterinária, v.15, p.88-93, 2008.

MADEN, M. et al. Specific enzyme activities in bronchoalveolar lavage fluid as an aid to diagnosis of tracheobronchitis and 
bronchopneumonia in dogs. Research in Veterinary Science, v.71, n.2, p.141-145, 2001. doi:10.1053/rvsc.2001.0503.

MAZAN, M.R. Inflammatory airway disease in the horse. In: AAEP FOCUS ON UPPER AND LOWER RESPIRATORY DISEASES, 2010, Salt Lake City, UT. Proceedings... Salt Lake City: AAEP, 2010. p.100-106.

McGORUM, B.C. et al. Evaluation of urea and albumen as endogenous markers of dilution of equine bronchoalveolar lavage fluid. Research in Veterinary Science, v.55, n.1, p.52-56, 1993. doi: 10.1016/003452889390033C

McGORUM B.C. et al. Exame clínico do trato respiratório. In: RADOSTITS O.M. et al. (Eds.). Exame clínico e diagnóstico em veterinária. Rio de Janeiro: Guanabara Koogan, 2002. Cap.16, p.231-269.

SANCHEZ, N.M.R. et al. Alkaline phosphatase isoenzymes in serum and bronchoalveolar lavage from patients with bronchopulmonary disease. Anales de Medicina Interna, v.17, n.4, p.182-185, 2000.

SILVA K.M. et al. Estimativa do volume de fluido epitelial pulmonar recuperado no lavado broncoalveolar no equino. Ciência Animal Brasileira, v.11, n.3, p.576-581, 2010. Disponível em: $<$ http://www.revistas.ufg.br/index.php/vet/article/view/5137>. Acesso em: 14 jan.2011. doi: 10.5216/cab.v11i3.5137.

VAN ERCK, E. Sampling the respiratory tract: techniques and interpretation. In: GENEVA CONGRESS ON EQUINE MEDICINE AND SURGERY, 11., 2009, Westergren, Belgium. Proceedings... Westergren: IVIS, 2009. Disponível em: <http:// www.ivis.org >. Acesso em: 19 jul.2011. p.45-47.

VIEL, L. Structural-functional correlations of the lung in horses with small airway disease. In: INTERNATIONAL SYMPOSIUM ON LUNG FUNCTION AND RESPIRATORY DISEASES IN THE HORSE, 1986, Hannover. Proceedings... Calw: Hippiatrika, 1986. p.41-45. 